\title{
WEIGHTED INEQUALITIES FOR ONE-SIDED MAXIMAL FUNCTIONS
}

\section{F. J. MARTÍN-REYES, P. ORTEGA SALVADOR, AND A. DE LA TORRE}

Abstract. Let $M_{g}^{+}$be the maximal operator defined by

$$
M_{g}^{+} f(x)=\sup _{h>0}\left(\int_{x}^{x+h}|f(t)| g(t) d t\right)\left(\int_{x}^{x+h} g(t) d t\right)^{-1},
$$

where $g$ is a positive locally integrable function on $\mathbf{R}$. We characterize the pairs of nonnegative functions $(u, v)$ for which $M_{g}^{+}$applies $L^{p}(v)$ in $L^{p}(u)$ or in weak- $L^{p}(u)$. Our results generalize Sawyer's (case $g=1$ ) but our proofs are different and we do not use Hardy's inequalities, which makes the proofs of the inequalities self-contained.

\section{INTRODUCTION}

In this paper we will study the operator $M_{g}^{+}$acting on measurable real functions on $\mathbf{R}$ defined by

$$
M_{g}^{+} f(x)=\sup _{h>0} \int_{x}^{x+h}|f(t)| g(t) d t\left(\int_{x}^{x+h} g(t) d t\right)^{-1},
$$

where $g$ is a locally integrable and positive function. If $g=1$ we obtain the one-sided Hardy-Littlewood maximal operator which has been studied by Sawyer [7].

We will characterize the pairs of weights $(u, v)$ such that $M_{g}^{+}$is of weak and strong type $(p, p)$ with respect to the measures $v d x$ and $u d x$. Our results include Sawyer's as particular cases, but with different proofs. The proof of the theorem about the weak type $(p, p)(p>1)$ is adapted from [1]. On the other hand, the proof of the theorem about the strong type $(p, p)$ is simpler than the corresponding one in [7] (our proof follows the pattern of the proof in [6]) and besides we do not use Hardy's inequalities which makes the proofs of the inequalities self-contained. We also include the weak type $(1,1)$ that is not

Received by the editors December 1, 1987.

1980 Mathematics Subject Classification (1985 Revision). Primary 42B25.

Key words and phrases. One-sided maximal functions, weighted inequalities, weights.

The research of the first and third author has been supported by C.A.I.C.Y.T. Grant (PB850434). 
studied in [7]. Finally we give several results about the good weights for $M_{g}^{+}$ such as relations with Muckenhoupt's classes, factorization, and extrapolation.

\section{NotATION AND MAIN RESUltS}

Throughout this paper, $g$ will be a positive locally integrable function and $C$ a positive constant not necessarily the same at each occurrence. If $p>1$, then its conjugate exponent will be denoted by $p^{\prime}$, and for a Lebesgue measurable set $A, \chi_{A}$ will be its characteristic function and $|A|$ its measure.

We will say that a pair of nonnegative functions $(u, v)$ satisfies condition $A_{p}^{+}(g), p>1$, if there exists a constant $C>0$ such that for every $y, x, b$ with $y \leq x \leq b$,

$$
\int_{y}^{x} u\left(\int_{x}^{b} g^{p^{\prime}} \sigma\right)^{p-1} \leq C\left(\int_{y}^{b} g\right)^{p},
$$

where $\sigma=v^{-1 / p-1}$ (as usual, we consider $0 \cdot \infty=0$ ).

Condition $A_{1}^{+}(g)$ is given by

$$
M_{g}^{-}\left(g^{-1} u\right) \leq C g^{-1} v \text { a.e., }
$$

where $M_{g}^{-}$is the left maximal operator defined in the obvious way.

A pair of nonnegative functions $(u, v)$ satisfies condition $S_{p}^{+}(g), p>1$, if there exists a constant $C>0$ such that for every interval $I=(a, b)$ with $\int_{(-\infty, a)} u>0$,

$$
\int_{a}^{b}\left(M_{g}^{+}\left(\chi_{I} g^{1 / p-1} \sigma\right)\right)^{p} u \leq C \int_{a}^{b} g^{p^{\prime}} \sigma<\infty .
$$

Our main results are the following three theorems.

Theorem 1. $M_{g}^{+}$is of weak type $(p, p), p>1$, with respect to the measures $v d x$ and $u d x$ if and only if $(u, v)$ satisfies $A_{p}^{+}(g)$.

Theorem 2. $M_{g}^{+}$is of strong type $(p, p), p \geq 1$, from $L^{p}(v)$ to $L^{p}(u)$ if and only if $(u, v)$ satisfies $S_{p}^{+}(g)$.

Theorem 3. If $u=v$ and $p>1, A_{p}^{+}(g)$ and $S_{p}^{+}(g)$ are equivalent conditions, that is, the weak type $(p, p)$ is equivalent to the strong type $(p, p)$.

\section{Proof OF THEOREM 1 FOR $p=1$}

We will need two lemmas:

Lemma 1. Let $w$ be a positive increasing function defined on $I=[a, b]$ (i.e., $s \leq t$ implies $w(s) \leq w(t))$. Let $f$ be a positive function on $I$. Suppose for some positive number $\lambda$

$$
\int_{t}^{b} g f \geq \lambda \int_{t}^{b} g \quad \text { for every } t \in I .
$$

Then $\lambda \int_{a}^{b} g w \leq \int_{a}^{b} g f w$. 
Proof of Lemma 1. Let $B>1$. Let

$$
\begin{aligned}
E=\{t \in[a, b]: \lambda & \int_{a}^{b} g w \\
& \left.\leq \lambda \int_{a}^{t} g w+B w(t)\left[\lambda \int_{t}^{b} g-\int_{t}^{b} f g\right]+B \int_{t}^{b} f g w\right\} .
\end{aligned}
$$

Let $\tau=\inf E$ ( $E$ is nonempty). We claim that $\tau=a$. If $a<\tau$, let $\eta \in(a, \tau)$ such that $B w(\eta) \geq \operatorname{ess} \sup \{w(t): a<t \leq \tau\}$. We will prove that $\eta \in E$, which will contradict that $\tau=\inf E$. Since $\tau \in E$ we have

$$
\lambda \int_{a}^{b} g w \leq \lambda \int_{a}^{\tau} g w+B w(\tau)\left[\lambda \int_{\tau}^{b} g-\int_{\tau}^{b} f g\right]+B \int_{\tau}^{b} f g w .
$$

Now, the fact that $w$ is increasing, the assumptions of the lemma and $\eta<\tau$ give

$$
\begin{aligned}
\lambda \int_{a}^{b} g w \leq & \lambda \int_{a}^{\eta} g w+\lambda \int_{\eta}^{\tau} g w+B w(\eta)\left[\lambda \int_{\tau}^{b} g-\int_{\tau}^{b} f g\right] \\
& +B \int_{\eta}^{b} f g w-B \int_{\eta}^{\tau} f g w .
\end{aligned}
$$

If we use again that $w$ is increasing and the election of $\eta$, we obtain

$$
\begin{aligned}
\lambda \int_{a}^{b} g w \leq & \lambda \int_{a}^{\eta} g w+B w(\eta)\left[\lambda \int_{\tau}^{b} g-\int_{\tau}^{b} f g\right] \\
& +B \int_{\eta}^{b} f g w+B w(\eta)\left[\lambda \int_{\eta}^{\tau} g-\int_{\eta}^{\tau} f g\right] \\
= & \lambda \int_{a}^{\eta} g w+B w(\eta)\left[\lambda \int_{\eta}^{b} g-\int_{\eta}^{b} f g\right]+B \int_{\eta}^{b} f g w .
\end{aligned}
$$

This means that $\eta \in E$, a contradiction. Hence, $\tau=a$ and then $a \in E$, that is

$$
\lambda \int_{a}^{b} g w \leq B w(a)\left[\lambda \int_{a}^{b} g-\int_{a}^{b} f g\right]+B \int_{a}^{b} f g w .
$$

Since the expression in brackets is nonpositive, we obtain $\lambda \int_{a}^{b} g w \leq B \int_{a}^{b} f g w$. Letting $B$ tend to 1 , we have the result.

Lemma 2. If $(u, v)$ satisfies $A_{1}^{+}(g)$ and $[a, b]$ is an interval, then there exists an increasing function $w$ on $[a, b]$ such that

(i) $w(s) \leq C g^{-1}(s) v(s)$ a.e. $s \in[a, b]$.

(ii) $\int_{a}^{b} u \leq \int_{a}^{b} g w$.

Proof of Lermma 2. Let $G(y)=M_{g}^{-}\left(g^{-1} u \chi_{[a, b]}\right)(y)$. The function $G$ is lower semicontinuous and finite a.e. by $A_{1}^{+}(g)$. 
Let $w(x)=\min _{x \leq y \leq b} G(y)$. It is obvious that $w$ is increasing and verifies (i). To see (ii), let $0<B<1$ and

$$
A=\left\{t \in[a, b]: \int_{y}^{b} g w \geq B \int_{y}^{b} u \text { for every } y \in[t, b]\right\} .
$$

It is clear that $A$ is a closed interval $[\tau, b]$. We will prove that $\tau=a$.

Suppose $\tau>a$. Since $G$ is lower semicontinuous, there exists $\delta>0$ such that $G(x) \geq B G(\tau)$ if $x \in[\tau-\delta, \tau)$. For such an $x$,

$$
\begin{aligned}
w(x) & =\min _{x \leq y \leq b} G(y)=\min \left\{\min _{x \leq y \leq \tau} G(y), \min _{\tau \leq y \leq b} G(y)\right\} \\
& \geq \min \{B G(\tau), w(\tau)\} \geq B w(\tau) .
\end{aligned}
$$

By the definition of $w$, there exists $\gamma$ with $\tau \leq \gamma \leq b$ such that $w(\tau)=$ $G(\gamma)$. For every $x \in[\tau, \gamma], w(x)=w(\tau)=G(\gamma)$, and for every $x \in[\tau-$ $\delta, \tau), w(x) \geq B w(\tau)=B G(\gamma)$. Therefore, if $x \in[\tau-\delta, \gamma]$ then $w(x) \geq$ $B G(\gamma)$. Hence

$$
\int_{x}^{\gamma} g w \geq B G(\gamma) \int_{x}^{\gamma} g \geq B \int_{x}^{\gamma} u \text { for every } x \in[\tau-\delta, \gamma] .
$$

This means that $\tau-\delta \in A$, which contradicts that $\tau$ is the infimum of $A$. Therefore $\tau=a$ and then $\int_{a}^{b} g w \geq B \int_{a}^{b} u$. Letting $B$ tend to 1 the proof is finished.

Now, it is easy to prove that $A_{1}^{+}(g)$ is sufficient for the weak $(1,1)$ inequality. Let $f$ be a positive function with support bounded from above, and let $\lambda, N>$ 0 . Let $O_{\lambda, N}=(-N, \infty) \cap\left\{x: M_{g}^{+} f(x)>\lambda\right\} . O_{\lambda, N}$ is a bounded open set and therefore there exists a sequence of maximal pairwise disjoint finite intervals $\left\{\left(a_{j}, b_{j}\right)\right\}$ such that $O_{\lambda, N}=\bigcup\left(a_{j}, b_{j}\right)$ and $\int_{x}^{b_{j}} f g \geq \lambda \int_{x}^{b_{j}} g$ for every $x \in$ $\left(a_{j}, b_{j}\right)$. For each $j$, by Lemma 2 , there exists an increasing function $w_{j}$ on $\left[a_{j}, b_{j}\right]$ such that

$$
w_{j}(t) \leq C g^{-1}(t) v(t) \quad \text { a.e. } t \in\left[a_{j}, b_{j}\right]
$$

and

$$
\int_{a_{j}}^{b_{j}} u \leq \int_{a_{j}}^{b_{j}} g w_{j}
$$

If we apply Lemma 1 to each $w_{j}$, we obtain

$$
\lambda \int_{a_{j}}^{b_{j}} g w_{j} \leq \int_{a_{j}}^{b_{j}} g f w_{j} .
$$

Now (3.2), (3.3), and (3.1) give

$$
\begin{aligned}
\int_{O_{\lambda, N}} u & =\sum_{j} \int_{a_{j}}^{b_{j}} u \leq \sum_{j} \int_{a_{j}}^{b_{j}} g w_{j} \\
& \leq \lambda^{-1} \sum_{j} \int_{a_{j}}^{b_{j}} g f w_{j} \leq C \lambda^{-1} \sum_{j} \int_{a_{j}}^{b_{j}} f v=C \lambda^{-1} \int_{O_{\lambda, N}} f v .
\end{aligned}
$$


Letting $N$ tend to infinity we obtain $\int_{\left\{x ; M_{g}^{+} f(x)>\lambda\right\}} u \leq C \lambda^{-1} \int_{-\infty}^{+\infty} f v$.

Conversely, let us suppose that $M_{g}^{+}$is of weak type $(1,1)$ with respect to the measures $v d x$ and $u d x$. For every natural number $N$ we consider the set $E_{N}=\left\{x: g^{-1}(x) v(x) \leq N\right\}$ and the function $v_{N}=v \chi_{E_{N}}$. Let $F_{N}$ and $H_{N}$ be the Lebesgue sets of $g^{-1} v_{N}$ and $\chi_{E_{N}}$ respectively. It is clear that if $F=\bigcap_{N} F_{N} \cap H_{N}$ then $|\mathbf{R}-F|=0$. Let $x$ be in $F$, and let $\delta, \varepsilon>0$ such that $\int_{x-\delta}^{x+\varepsilon} g \leq 2 \int_{x-\delta}^{x} g$. Now consider $N$ with $g^{-1}(x) v(x) \leq N$. If $f_{N}=g^{-1} \chi_{E_{N} \cap(x, x+\varepsilon)}$ and $y \in(x-\delta, x)$ then

$$
M_{g}^{+} f_{N}(y) \geq \int_{x}^{x+\varepsilon} \chi_{E_{N}}\left(2 \int_{x-\delta}^{x} g\right)^{-1} .
$$

Therefore, by the weak type inequality,

$$
\int_{x-\delta}^{x} u \leq 2 C\left(\int_{x-\delta}^{x} g\right)\left(\int_{x}^{x+\varepsilon} g^{-1} v_{N}\right)\left(\int_{x}^{x+\varepsilon} \chi_{E_{N}}\right)^{-1} .
$$

If we let $\varepsilon$ tend to zero and then $N$ to infinity we get

$$
\int_{x-\delta}^{x} u \leq 2 C\left(\int_{x-\delta}^{x} g\right)\left(g^{-1} v\right)(x) .
$$

Since $\delta$ is an arbitrary positive number we obtain $M_{g}^{-} u(x) \leq 2 C\left(g^{-1} v\right)(x)$ for all $x$ in $F$ and thus for almost every $x$ in $\mathbf{R}$.

\section{Proof of Theorem 1 for $p>1$}

Suppose that $(u, v)$ satisfies $A_{p}^{+}(g)$ and $\int_{(-\infty, b)} g=\infty$ for every $b$ in $\mathbf{R}$. Then if $y \leq x \leq b$

$$
\left(\int_{y}^{x} u\right)\left(\int_{x}^{b} g^{p^{\prime}} \sigma\right)^{p-1} \leq C\left(\int_{y}^{b} g\right)^{p}
$$

with $C$ independent of $x, y$, and $b$. Let $\alpha>0$. Multiplying both sides of (4.1) by $g(y)\left(\int_{y}^{b} g\right)^{-p-\alpha-1}$ and integrating with respect to $y$ on $(-\infty, x)$ we get

$$
\begin{gathered}
\left(\int_{x}^{b} g^{p^{\prime}} \sigma\right)^{p-1} \int_{-\infty}^{x} g(y)\left(\int_{y}^{x} u\right)\left(\int_{y}^{b} g\right)^{-p-\alpha-1} d y \\
\leq C \int_{-\infty}^{x} g(y)\left(\int_{y}^{b} g\right)^{-\alpha-1} d y
\end{gathered}
$$

for every $x$. Computing the right-hand side of (4.2), we obtain

$$
\left(\int_{x}^{b} g^{p^{\prime}} \sigma\right)^{p-1} \int_{-\infty}^{x} g(y)\left(\int_{y}^{x} u\right)\left(\int_{y}^{b} g\right)^{-p-\alpha-1} d y \leq C \alpha^{-1}\left(\int_{x}^{b} g\right)^{-\alpha} .
$$


Besides

$$
\begin{array}{rl}
\int_{-\infty}^{x} & g(y)\left(\int_{y}^{x} u(t) d t\right)\left(\int_{y}^{b} g\right)^{-p-\alpha-1} d y \\
& =\int_{-\infty}^{x} u(t)\left(\int_{-\infty}^{t} g(y)\left(\int_{y}^{b} g\right)^{-p-\alpha-1} d y\right) d t \\
& =\int_{-\infty}^{x} u(t)(p+\alpha)^{-1}\left(\int_{t}^{b} g\right)^{-p-\alpha} d t
\end{array}
$$

(4.3) and (4.4) give

$$
\left(\int_{x}^{b} g^{p^{\prime}} \sigma\right)^{p-1} \int_{-\infty}^{x} u(t)\left(\int_{t}^{b} g\right)^{-p-\alpha} d t \leq C(p+\alpha) \alpha^{-1}\left(\int_{x}^{b} g\right)^{-\alpha} .
$$

It is interesting to note that (4.5) holds even if $g^{p^{\prime}} \sigma$ is not locally integrable, since $A_{p}^{+}(g)$ implies that if the integral of $g^{p^{\prime}} \sigma$ on $\left[x_{1}, x_{2}\right]$ is infinite then $u(t)=0$ for a.e. $t<x_{1}$.

Let $f$ be a positive function with support bounded from above. For $\lambda>0$ and natural $N$, let $O_{\lambda, N}=\left\{x: M_{g}^{+} f(x)>\lambda\right\} \cap(-N, \infty)$. Let $(a, b)$ be a connected component of $O_{\lambda}$. We have

$$
\lambda \int_{x}^{b} g \leq \int_{x}^{b} f g \quad \text { for every } x \text { in }(a, b) .
$$

Let $A=\left\{x \in[a, b]: \int_{x}^{b} g^{p^{\prime}} \sigma=\infty\right\}$. If $A \neq \varnothing$, let $x_{0}=\sup A$; if $A=\varnothing$, let $x_{0}=a$. Then $\int_{x}^{b} g^{p^{\prime}} \sigma<\infty$ for every $x>x_{0}$ and it follows from $A_{p}^{+}(g)$ that $u(x)=0$ a.e. in $\left[a, x_{0}\right]$. Thus $\int_{a}^{b} u=\int_{x_{0}}^{b} u$.

Let $H$ and $h$ be the functions defined on $(a, b)$ by

$$
H(x)=\int_{a}^{x} u(t)\left(\int_{t}^{b} g\right)^{-p-\alpha} d t \quad \text { and } \quad h(x)=\left(\int_{x}^{b} g^{p^{\prime}} \sigma\right)^{1 / p^{\prime}}
$$

It is clear that $H(x)=0$ and $h(x)=\infty$ if $x<x_{0}$ and from (4.5) we get

$$
(h(x))^{p} H(x) \leq C(p+\alpha) \alpha^{-1}\left(\int_{x}^{b} g\right)^{-\alpha} \quad \text { for every } x \in[a, b] .
$$

On the other hand we have

$$
\int_{a}^{b} u(x) d x=\int_{x_{0}}^{b} H^{\prime}(x)\left(\int_{x}^{b} g\right)^{p+\alpha} d x
$$


Integration by parts in the right-hand side of (4.8) gives

$$
\begin{aligned}
\int_{a}^{b} u(x) d x & =(p+\alpha) \int_{x_{0}}^{b} H(x) g(x)\left(\int_{x}^{b} g\right)^{p+\alpha-1} d x \\
& \leq C(p+\alpha)^{2} \alpha^{-1} \int_{x_{0}}^{b} h^{-p}(x) g(x)\left(\int_{x}^{b} g\right)^{p-1} d x
\end{aligned}
$$

by (4.7). Again integration by part gives

$$
\begin{aligned}
\int_{a}^{b} u \leq C(p+\alpha)^{2} \alpha^{-1} & \left(p^{-1} h^{-p}\left(x_{0}\right)\left(\int_{x_{0}}^{b} g\right)^{p}\right. \\
& \left.-\int_{x_{0}}^{b} h^{\prime}(x) h^{-p-1}(x)\left(\int_{x}^{b} g\right)^{p} d x\right) .
\end{aligned}
$$

On the other hand, if we raise both sides of (4.6) to the $p$ th power and apply Hölder's inequality with exponents $p$ and $p^{\prime}$, after introducing suitable factors, we obtain

$$
\begin{aligned}
\lambda^{p} & \leq\left(\int_{x}^{b} g\right)^{-p}\left(\int_{x}^{b} g f\right)^{p} \\
& \leq\left(\int_{x}^{b} g\right)^{-p}\left(\int_{x}^{b} f^{p} h v\right)\left(\int_{x}^{b} g^{p^{\prime}} \sigma h^{-p^{\prime} / p}\right)^{p / p^{\prime}} .
\end{aligned}
$$

Computing the last integral of the above inequality gives us

$$
\begin{aligned}
\int_{x}^{b} g^{p^{\prime}} \sigma h^{-p^{\prime} / p} & =\int_{x}^{b} g^{p^{\prime}}(t) \sigma(t)\left(\int_{t}^{b} g^{p^{\prime}} \sigma\right)^{-1 / p} d t \\
& =p^{\prime}\left(\int_{x}^{b} g^{p^{\prime}} \sigma\right)^{1 / p^{\prime}}=p^{\prime} h(x) .
\end{aligned}
$$

Then (4.11) becomes

$$
\left(\int_{x}^{b} g\right)^{p} \leq \lambda^{-p} p^{p-1}\left(\int_{x}^{b} f^{p} h v\right) h^{p-1}(x) .
$$

If we set $x=x_{0}$ in (4.13), we obtain an inequality that allows us to majorize the first addend of the right-hand side in (4.10), i.e.,

$$
h^{-p}\left(x_{0}\right)\left(\int_{x_{0}}^{b} g\right)^{p} \leq \lambda^{-p} p^{p-1}\left(\int_{x_{0}}^{b} f^{p} h v\right) h^{-1}\left(x_{0}\right) .
$$

To majorize the second addend we will use (4.13), the positivity of $-h^{\prime}$, and 
integration by parts:

$$
\begin{aligned}
& -\int_{x_{0}}^{b} h^{-p-1}(x) h^{\prime}(x)\left(\int_{x}^{b} g\right)^{p} d x \\
& \leq-\lambda^{-p} p^{\prime p-1} \int_{x_{0}}^{b} h^{-2}(x) h^{\prime}(x)\left(\int_{x}^{b} f^{p} h v\right) d x \\
& \quad=\lambda^{-p} p^{p-1}\left(-h^{-1}\left(x_{0}\right) \int_{x_{0}}^{b} f^{p} h v+\int_{x_{0}}^{b} f^{p} v\right) .
\end{aligned}
$$

Finally, (4.14), (4.15), and (4.10) give

$$
\int_{a}^{b} u \leq C \lambda^{-p}(p+\alpha)^{2} p^{p-1} \alpha^{-1} \int_{x_{0}}^{b} f^{p} v \leq C \lambda^{-p}(p+\alpha)^{2} p^{\prime p-1} \alpha^{-1} \int_{a}^{b} f^{p} v
$$

We have proved that

$$
\int_{O_{\lambda, N}} u \leq C \lambda^{-p} \int_{O_{\lambda, N}} f^{p} v
$$

Letting $N$ tend to infinity we obtain the weak inequality.

Everything we have just done is based on the assumption $\int_{(-\infty, b)} g=\infty$ for every $b$. If it is not true we define $g_{n}=g$ if $x \geq-n$ and $g_{n}=\max \{g, 1\}$ if $x<-n$, for every natural $n$. Every $g_{n}$ verifies $\int_{-\infty}^{b} g_{n}=\infty$ for every $b$, and since $(u, v) \in A_{p}^{+}(g)$ we have that $\left(u, g^{-p} g_{n}^{p} v\right)$ satisfies $A_{p}^{+}\left(g_{n}\right)$. Then, by what we have already shown

$$
\int_{\left\{x: M_{g_{n}}^{+} f(x)>\lambda\right\}} u \leq C \lambda^{-p} \int_{-\infty}^{+\infty}|f|^{p} g_{n}^{p} g^{-p} v
$$

for every $f$, where $C$ depends only on the constant of the $A_{p}^{+}(g)$ condition. Now if we apply (4.17) to the functions $f \chi_{(-n, \infty)}$ we have

$$
\int_{\left\{x \geq-n: M_{g}^{+} f(x)>\lambda\right\}} u \leq C \lambda^{-p} \int_{-n}^{+\infty}|f|^{p} v \text {. }
$$

Letting $n$ tend to infinity we obtain the weak type inequality.

Conversely, suppose that $M_{g}^{+}$is of weak type $(p, p)$ with respect to the measures $v d x$ and $u d x$. Let $x, y, b$ be given with $x \leq y \leq b$. For every natural $n$, let $h_{n}=g^{p^{\prime}} \sigma \chi_{\left\{x: g^{p^{\prime}} \sigma(x)<n\right\}} \cdot\left\{h_{n}\right\}$ is an increasing sequence with limit $g^{p^{\prime}} \sigma$. Let $f=\chi_{(y, b)}\left(g^{-1} v\right)^{-1 / p-1} \chi_{\left\{x: g^{p^{\prime}} \sigma<n\right\}}$ and $B_{n}=\int_{y}^{b} h_{n}\left(\int_{x}^{b} g\right)^{-1}$. If $z \in[x, y]$ we have $M_{g}^{+} f(z) \geq B_{n}$. Then, the weak type inequality gives

$$
\int_{x}^{y} u \leq C B_{n}^{-p} \int_{y}^{b} h_{n}
$$

or equivalently

$$
\left(\int_{x}^{y} u\right)\left(\int_{y}^{b} h_{n}\right)^{p-1} \leq C\left(\int_{x}^{b} g\right)^{p}
$$


Now the $A_{p}^{+}(g)$ condition follows from the monotone convergence theorem.

\section{Proof of Theorem 2}

The necessity of $S_{p}^{+}(g)$ for the two-norm inequality is trivial. For the converse it will suffice to prove the strong type inequality for bounded positive $f$ in $L^{p}(v)$ with support bounded from above.

Let $N$ be a positive integer. For $k>0$ let

$$
O_{k}=\left\{x \in \mathbf{R}: M_{g}^{+} f(x)>2^{k}\right\} \cap(-N,+\infty) .
$$

Each $O_{k}$ is an open set and, therefore, there exists a sequence $\left\{I_{j k}\right\}_{j}$ of open pairwise disjoint intervals with $O_{k}=\bigcup_{j} I_{j k}$ and such that

$$
\int_{x}^{b_{j k}} g f \geq 2^{k} \int_{x}^{b_{j k}} g \quad \text { for every } x \in I_{j k}=\left(a_{j k}, b_{j k}\right) .
$$

It is clear that $\sup _{j, k}\left|I_{j k}\right|<\infty$. For every $j$ and $k$ let $A_{j k}=\left\{x \in I_{j k}\right.$ : $\left.\int_{x}^{b_{j k}} g^{p^{\prime}} \sigma=\infty\right\}$. If $A_{j k} \neq \varnothing$, let $x_{j k}=\sup A$; if $A_{j k}=\varnothing$, let $x_{j k}=a_{j k}$. It is clear that $\int_{x}^{b_{j k}} g^{p^{\prime}} \sigma<\infty$ if $x>x_{j k}$ and $u=0$ a.e. $x$ in $\left(a_{j k}, x_{j k}\right)$ by $S_{p}^{+}(g)$. For every $j$ and $k$ let

$$
E_{j k}=I_{j k} \cap\left\{x: M_{g}^{+} f(x) \leq 2^{k+1}\right\} \quad \text { and } \quad F_{j k}=\left(x_{j k}, b_{j k}\right) \cap E_{j k} .
$$

The sets $E_{j k}$ are pairwise disjoint and for every $k$

$$
\bigcup_{j} E_{j k}=\left\{x: 2^{k}<M_{g}^{+} f(x) \leq 2^{k+1}\right\} \cap(-N, \infty) .
$$

Then

$$
\begin{aligned}
\int_{-N}^{+\infty}\left(M_{g}^{+} f\right)^{p} u & =\sum_{k} \int_{(-N,+\infty) \cap\left\{x: 2^{k}<M_{g}^{+} f(x) \leq 2^{k+1}\right\}}\left(M_{g}^{+} f\right)^{p} u \\
& =\sum_{k, j} \int_{E_{j k}}\left(M_{g}^{+} f\right)^{p} u=\sum_{k, j} \int_{F_{j k}}\left(M_{g}^{+} f\right)^{p} u .
\end{aligned}
$$

By the definition of $F_{j k}$ and by (5.1) we have that the last term is smaller than or equal to

$$
2^{p} \sum_{k, j} \int_{F_{j k}} u(x)\left(\int_{x}^{b_{j k}} f g\right)^{p}\left(\int_{x}^{b_{j k}} g\right)^{-p} d x
$$

Therefore

$$
\begin{aligned}
\int_{-N}^{+\infty}\left(M_{g}^{+} f\right)^{p} u \leq & 2^{p} \sum_{k, j} \int_{F_{j k}} u(x)\left(\int_{x}^{b_{j k}} f g\right)^{p}\left(\int_{x}^{b_{j k}} g^{p^{\prime}} \sigma\right)^{-p} \\
& \times\left(\int_{x}^{b_{j k}} g^{p^{\prime}} \sigma\right)^{p}\left(\int_{x}^{b_{j k}} g\right)^{-p} d x
\end{aligned}
$$


Let $X=\mathbf{Z} \times \mathbf{Z} \times \mathbf{R}$ and let $\omega$ be the product measure $\nu \times \nu \times m$ where $\nu$ is the counting measure on $\mathbf{Z}$ and $m$ is the Lebesgue measure on $\mathbf{R}$. Let $\varphi$ be the real function defined on $X$ by

$$
\varphi(j, k, x)=\chi_{F_{j k}}(x) u(x)\left(\int_{x}^{b_{j k}} g^{p^{\prime}} \sigma\right)^{p}\left(\int_{x}^{b_{j k}} g\right)^{-p}
$$

and let $T$ be the linear operator

$$
T h(j, k, x)=\int_{x}^{b_{j k}} h g^{p^{\prime}} \sigma\left(\int_{x}^{b_{j k}} g^{p^{\prime}} \sigma\right)^{-1} .
$$

With these notations inequality (5.3) can be written in the following way:

$$
\int_{-N}^{+\infty}\left(M_{g}^{+} f\right)^{p} u \leq 2^{p} \int_{X}\left[T\left(f\left(g^{-1} v\right)^{1 / p-1}\right)\right]^{p} \varphi d \omega .
$$

If we prove that the operator $T$ is bounded from $L^{p}\left(g^{p^{\prime}} \sigma d x\right)$ to $L^{p}(X, \varphi d \omega)$, we will get

$$
\int_{-N}^{+\infty}\left(M_{g}^{+} f\right)^{p} u \leq C 2^{p} \int_{-\infty}^{+\infty}\left(f\left(g^{-1} v\right)^{1 / p-1}\right)^{p} g^{p^{\prime}} \sigma=C 2^{p} \int_{-\infty}^{+\infty} f^{p} v
$$

and, letting $N$ tend to infinity, the proof will be finished.

To prove the boundedness of $T$ we observe that it is obviously bounded in $L^{\infty}$ and by Marcinkiewickz's interpolation theorem it will be enough to prove the weak type $(1,1)$, i.e., $\int_{\{(j, k, x) \in X: T h(j, k, x)>\lambda\}} \varphi d \omega \leq C \lambda^{-1} \int_{-\infty}^{+\infty} h g^{p^{\prime}} \sigma$ with $C$ the constant of condition (2.3).

Let $A_{j k}(\lambda)=F_{j k} \cap\{x: T h(j, k, x)>\lambda\}$. The sets $A_{j k}$ are pairwise disjoint. For each pair $j, k$, let $s_{j k}(\lambda)=\inf A_{j k}(\lambda)$ and $J_{j k}=J_{j k}(\lambda)=\left[s_{j k}(\lambda), b_{j k}\right)$. If we pick up two of these intervals $J_{j k}$ and $J_{l m}$, then they are either disjoint or one of them is contained in the other. Also it is clear that each $J_{j k}$ verifies

$$
\int_{J_{j k}} h g^{p^{\prime}} \sigma \geq \lambda \int_{J_{j k}} g^{p^{\prime}} \sigma
$$

Let $\left\{J_{i}\right\}$ be the maximal elements of the family $\left\{J_{j k}\right\}$. These maximal elements exist since the intervals $J_{j k}$ have uniformly bounded lengths. Also the 
WEIGHTED INEQUALITIES FOR ONE-SIDED MAXIMAL FUNCTIONS

527 intervals $J_{i}$ verify $(5.5)$. Then

$$
\begin{aligned}
& \int_{\{(j, k, x): T h(j, k, x)>\lambda\}} \varphi(j, k, x) d \omega \\
& \quad=\sum_{k, j} \int_{A_{j k}(\lambda)} u(x)\left(\int_{x}^{b_{j k}} g^{p^{\prime}} \sigma\right)^{p}\left(\int_{x}^{b_{j k}} g\right)^{-p} d x \\
& \quad \leq \sum_{i} \sum_{\left\{(k, j): J_{i} \supset J_{j k}\right\}} \int_{A_{j k}(\lambda)} u(x)\left(\int_{x}^{b_{j k}} g^{p^{\prime}} \sigma\right)^{p}\left(\int_{x}^{b_{j k}} g\right)^{-p} d x \\
& \leq \sum_{i} \int_{J_{i}}\left(M_{g}^{+}\left(\chi_{J_{i}} g^{1 / p-1} \sigma\right)(x)\right)^{p} u(x) d x \\
& \leq C \sum_{i} \int_{J_{i}} g^{p^{\prime}}(x) \sigma(x) d x \text { by }(2.3),
\end{aligned}
$$

and by (5.5) the last term is smaller than or equal to

$$
C \lambda^{-1} \sum_{i} \int_{J_{i}} h(x) g^{p^{\prime}}(x) \sigma(x) d x \leq C \lambda^{-1} \int_{-\infty}^{+\infty} h(x) g^{p^{\prime}}(x) \sigma(x) d x .
$$

This proves the weak $(1,1)$ inequality for $T$ and hence the proof of Theorem 2 is finished.

6. Proof of Theorem 3

Suppose that $u \in A_{p}^{+}(g)$. Let $I=(a, b)$ be an interval such that $\int_{-\infty}^{a} u>0$. This implies that $\int_{a}^{b} g^{p^{\prime}} \sigma<\infty$ where $\sigma=u^{-1 / p-1}$. Let $x \in I$ then there exist $h>0$ with $x+h \in I$ such that

$$
\frac{3}{4} M_{g}^{+}\left(\chi_{I} g^{1 / p-1} \sigma\right)(x) \leq \int_{x}^{x+h} g^{p^{\prime}} \sigma\left(\int_{x}^{x+h} g\right)^{-1} \text {. }
$$

For this $h$ there exists $t$ with $0<t<h$ such that $2 \int_{x}^{x+t} g=\int_{x}^{x+h} g$. This $t$ verifies

$$
\int_{x}^{x+t} g^{p^{\prime}} \sigma\left(\int_{x}^{x+t} g\right)^{-1} \leq M_{g}^{+}\left(\chi_{I} g^{1 / p-1} \sigma\right)(x) .
$$

(6.1) and (6.2) give

$$
M_{g}^{+}\left(\chi_{I} g^{1 / p-1} \sigma\right)(x) \leq 4 \int_{x+t}^{x+h} g^{p^{\prime}} \sigma\left(\int_{x}^{x+h} g\right)^{-1} .
$$

On the other hand, condition $A_{p}^{+}(g)$ for $u$ gives

$$
\int_{x+t}^{x+h} g^{p^{\prime}} \sigma\left(\int_{x}^{x+h} g\right)^{-1} \leq C\left(\int_{x}^{x+h} g\right)^{p^{\prime}-1}\left(\int_{x}^{x+t} u\right)^{1-p^{\prime}} .
$$


Now (6.3) together with (6.4) gives

$$
\begin{aligned}
M_{g}^{+}\left(\chi_{I} g^{1 / p-1} \sigma\right)(x) & \leq C\left(\int_{x}^{x+h} g\right)^{p^{\prime}-1}\left(\int_{x}^{x+t} u\right)^{1-p^{\prime}} \\
& \leq C\left(M_{u}^{+}\left(\chi_{I} g u^{-1}\right)(x)\right)^{p^{\prime}-1}
\end{aligned}
$$

Raising to $p$ and multiplying by $u(x)$, we get

$$
\left(M_{g}^{+}\left(\chi_{I} g^{1 / p-1} \sigma\right)(x)\right)^{p} u(x) \leq C\left(M_{u}^{+}\left(\chi_{I} g u^{-1}\right)(x)\right)^{p^{\prime}} u(x) .
$$

But $M_{u}^{+}$is bounded in $L^{p^{\prime}}(u)$, because $u \in A_{r}^{+}(u)$ for every $r>1$. Then

$$
\int_{I}\left(M_{g}^{+}\left(\chi_{I} g^{1 / p-1} \sigma\right)(x)\right)^{p} u(x) d x \leq C \int_{I} g^{p^{\prime}} \sigma .
$$

Therefore $u$ satisfies $S_{p}^{+}(g)$.

The fact that $S_{p}^{+}(g)$ implies $A_{p}^{+}(g)$ is a consequence of Theorems 1 and 2, not only for a weight, but for pairs of weights. However, we are going to give a direct proof. Suppose that the pair $(u, v)$ satisfies $S_{p}^{+}(g)$. Let $a, b$, and $c$ be real numbers with $a \leq b \leq c$. If the integral of $g^{p^{\prime}} \sigma$ on $[b, c]$ is equal to infinity, then, by $S_{p}^{+}(g), u(x)=0$ a.e. $x$ in $[a, b]$ and the inequality

$$
\int_{a}^{b} u\left(\int_{b}^{c} g^{p^{\prime}} \sigma\right)^{p-1} \leq C\left(\int_{a}^{c} g\right)^{p}
$$

is trivially satisfied.

Now suppose that the integral of $g^{p^{\prime}} \sigma$ over $[b, c]$ is finite. We define a possibly finite decreasing sequence by $x_{0}=b$ and $x_{k+1}$ the real number such that

$$
2^{k+1} \int_{b}^{c} g^{p^{\prime}} \sigma=\int_{x_{k+1}}^{c} g^{p^{\prime}} \sigma
$$

if

$$
\int_{-\infty}^{x_{k}} g^{p^{\prime}} \sigma \geq 2^{k} \int_{b}^{c} g^{p^{\prime}} \sigma
$$

otherwise the sequence finishes in $x_{k}$.

Suppose first that the sequence is finite and $x_{r}$ is its last term. If $r=0$, then

$$
\begin{aligned}
\int_{a}^{b} u(x)\left(\int_{x}^{c} g\right)^{-p}\left(\int_{b}^{c} g^{p^{\prime}} \sigma\right)^{p} d x & \leq \int_{a}^{b} u(x)\left(M_{g}^{+}\left(\chi_{(a, b)} g^{1 / p-1} \sigma\right)(x)\right)^{p} d x \\
& \leq C \int_{a}^{c} g^{p^{\prime}} \sigma \leq 2 C \int_{b}^{c} g^{p^{\prime}} \sigma .
\end{aligned}
$$

This implies trivially $A_{p}^{+}(g)$. 
If $r>0$, let $a^{\prime}<x_{r}$ and $a^{\prime}<a$. Then,

$$
\begin{aligned}
\int_{a}^{b} u(x) & \left(\int_{x}^{c} g\right)^{-p}\left(\int_{b}^{c} g^{p^{\prime}} \sigma\right)^{p} d x \\
\leq & 2^{-r p} \int_{a^{\prime}}^{x_{r}} u(x)\left(\int_{x}^{c} g\right)^{-p}\left(\int_{x_{r}}^{c} g^{p^{\prime}} \sigma\right)^{p} d x \\
& +\sum_{k=0}^{r-1} 2^{-k p} \int_{x_{k+1}}^{x_{k}} u(x)\left(\int_{x}^{c} g\right)^{-p}\left(\int_{x_{k}}^{c} g^{p^{\prime}} \sigma\right)^{p} d x \\
\leq & 2^{-r p} \int_{a^{\prime}}^{x_{r}} u(x)\left(M_{g}^{+}\left(\chi_{\left(a^{\prime}, c\right)} g^{1 / p-1} \sigma\right)(x)\right)^{p} d x \\
& +\sum_{k=0}^{r-1} 2^{-k p} \int_{x_{k+1}}^{x_{k}}\left(M_{g}^{+}\left(\chi_{\left(x_{k+1}, c\right)} g^{1 / p-1} \sigma\right)(x)\right)^{p} u(x) d x \\
\leq & C\left(\sum_{k=0}^{r} 2^{-k p+k+1}\right) \int_{b}^{c} g^{p^{\prime}} \sigma \leq C \int_{b}^{c} g^{p^{\prime}} \sigma .
\end{aligned}
$$

Finally, suppose that the sequence is infinite and let $d=\lim x_{k}$. If $d$ is finite, then $u=0$ a.e. in $(-\infty, d)$ by $S_{p}^{+}(g)$. So, whether $d$ is finite or not we have

$$
\int_{a}^{b} u(x)\left(\int_{x}^{c} g\right)^{-p}\left(\int_{b}^{c} g^{p^{\prime}} \sigma\right)^{p} d x \leq \int_{d}^{b} u(x)\left(\int_{x}^{c} g\right)^{-p}\left(\int_{b}^{c} g^{p^{\prime}} \sigma\right)^{p} d x .
$$

Using the reasoning above with sum from 0 to $\infty$ completes the proof.

\section{FURTHER RESUlts}

(A) Relations with Muckenhoupt's $A_{p}(g)$ classes and Sawyer's $S_{p}(g)$ classes. Consider the weighted two-sided Hardy-Littlewood maximal operator defined by

$$
M_{g} f(x)=\sup _{h, s>0}\left(\int_{x-s}^{x+h}|f| g\right)\left(\int_{x-s}^{x+h} g\right)^{-1} .
$$

It is clear that the following relation holds:

$$
\frac{1}{2}\left(M_{g}^{+}+M_{g}^{-}\right) \leq M_{g} \leq M_{g}^{+}+M_{g}^{-} .
$$

We have the following results for $M_{g}$ (see e.g. [5, 6]):

(i) Let $1 \leq p<\infty . M_{g}$ is of weak type $(p, p)$ with respect to the measures $v d x$ and $u d x$ if and only if the pair $(u, v)$ satisfies $A_{p}(g)$, i.e.

$A_{p}(g)$ : There exists $C>0$ such that

$$
\left(\int_{a}^{b} u\right)\left(\int_{a}^{b} g^{p^{\prime}} \sigma\right)^{p-1} \leq C\left(\int_{a}^{b} g\right)^{p}
$$

for every interval $(a, b)$ and $p>1$. 
$A_{1}(g)$ : There exists $C>0$ such that $M_{g}\left(g^{-1} u\right) \leq C g^{-1} v$ a.e.

(ii) Let $1<p<\infty . M_{g}$ is of strong type $(p, p)$ with respect to the measures $v d x$ and $u d x$ if and only if the pair $(u, v)$ satisfies $S_{p}(g)$, i.e.,

$S_{p}(g)$ : There exists $C>0$ such that for every interval $(a, b)$

$$
\int_{a}^{b}\left|M_{g}\left(\chi_{(a, b)} g^{1 / p-1} \sigma\right)\right|^{p} u \leq C \int_{a}^{b} g^{p^{\prime}} \sigma<\infty .
$$

Of course, if $u=v$ then $A_{p}(g)$ and $S_{p}(g)$ are equivalent conditions.

It follows from these results, our theorems, and (7.1) that $A_{p}(g)=A_{p}^{+}(g) \cap$ $A_{p}^{-}(g)(1 \leq p<\infty)$ and $S_{p}(g)=S_{p}^{+}(g) \cap S_{p}^{-}(g)(1<p<\infty)$. We will now give direct proofs of these equalities and so results (i) and (ii) will be consequences of the results in this paper.

Theorem 4. (a) $A_{p}(g)=A_{p}^{+}(g) \cap A_{p}^{-}(g)(1 \leq p<\infty)$.

(b) $S_{p}(g)=S_{p}^{+}(g) \cap S_{p}^{-}(g)(1<p<\infty)$.

Proof of Theorem 4. (a) For $p=1$ the equality is trivial by (7.1). Let $1<p$. Since it is clear that $A_{p}^{+}(g) \cap A_{p}^{-}(g) \supset A_{p}(g)$ we only have to prove $A_{p}(g) \supset$ $A_{p}^{+}(g) \cap A_{p}^{-}(g)$. Let $(u, v)$ be in $A_{p}^{+}(g) \cap A_{p}^{-}(g)$, let $a$ and $c$ be real numbers with $a \leq c$, let $N$ be a natural number, define $G_{N}(x)=\left(g^{p^{\prime}} \sigma\right)(x)$ if $\left(g^{p^{\prime}} \sigma\right)(x) \leq N$ and $G_{N}(x)=0$ otherwise. There exists $h$ such that

$$
\int_{a}^{c} G_{N}=2 \int_{a}^{h} G_{N}=2 \int_{h}^{c} G_{N} .
$$

Then

$$
\begin{aligned}
\int_{a}^{c} u\left(\int_{a}^{c} G_{N}\right)^{p-1} & =2^{p-1} \int_{a}^{h} u\left(\int_{h}^{c} G_{N}\right)^{p-1}+2^{p-1} \int_{h}^{c} u\left(\int_{a}^{h} G_{N}\right)^{p-1} \\
& \leq 2^{p} C\left(\int_{a}^{c} g\right)^{p} \quad \text { by } A_{p}^{+}(g) \text { and } A_{p}^{-}(g) .
\end{aligned}
$$

Letting $N$ tend to infinity we get $A_{p}(g)$.

Finally, (b) follows clearly from (7.1).

(B) Factorization. We will give here a result that generalizes the theorem of Coifman, Jones and Rubio de Francia [2] (see also [4]). As consequences, we will obtain the factorization of $A_{p}^{+}(g)$ and $A_{p}^{-}(g)$ weights.

Theorem 5. Let $F$ and $G$ be two sublinear operators acting on measurable functions of a measure space $(X, \mathfrak{M}, \mu)$. For $p>1$ let $W_{p}=\{w: F$ is bounded in $\left.L^{p}(w d \mu)\right\}$ and $U_{p}=\left\{u: G\right.$ is bounded in $\left.L^{p}(u d \mu)\right\}$. Let $g$ be a positive function, and let $W_{1}=\left\{w: G\left(g^{-1} w\right) \leq C g^{-1} w\right.$ a.e. $\}$ and $U_{1}=\left\{u: F\left(g^{-1} u\right) \leq\right.$ $C g^{-1}$ u a.e. $\}$. Then $g^{p-1} W_{1} U_{1}^{1-p} \supset W_{p} \cap g^{p} U_{p}^{1-p}$, i.e., if $w \in W_{p}$ and $g^{p^{\prime}} w^{-1 / p-1} \in U_{p}$ then there exist $w_{0} \in W_{1}$ and $u_{0} \in U_{1}$ such that $w=$ $g^{p-1} w_{0} u_{0}^{1-p}$. 
If $F=G$ and $g=1$ we obtain the above-mentioned result of Coifman, Jones, and Rubio de Francia.

Proof. This proof follows the proof of Theorem 5.2 in [4], with the obvious changes. Suppose $1<p \leq 2$. Let $w \in W_{p} \cap g^{p} U_{p}^{1-p}$. We have to find $v$ such that

(i) $v w \in W_{1}$, i.e., $G\left(g^{-1} v w\right) \leq C g^{-1} v w$ a.e.,

(ii) $g v^{1 / p-1} \in U_{1}$, i.e., $F\left(v^{1 / p-1}\right) \leq C v^{1 / p-1}$ a.e.

Let us define an operator $S$ by $S(u)=\left|G\left(g^{-1} u w\right)\right| w^{-1} g+\left(F\left(|u|^{1 / p-1}\right)\right)^{p-1}$. The operator $S$ is positive, sublinear, and bounded on $L^{p^{\prime}}(w)$. So, $S$ verifies the conditions of Lemma 5.1 in [4], and it ensures the existence of such a $v$. Then $w_{0}=v w$ and $u_{0}=g v^{1 / p-1}$.

Corollary 1. $w \in A_{p}^{+}(g)$ if and only if $w=g^{p-1} w_{0} w_{1}^{1-p}$ with $w_{0} \in A_{1}^{+}(g)$ and $w_{1} \in A_{1}^{-}(g)$.

Proof. If in Theorem 5, we take $F=M_{g}^{+}$and $G=M_{g}^{-}$, the classes of good weights are, respectively $W_{p}=A_{p}^{+}(g)$ and $U_{p}=A_{p}^{-}(g)$. Then Theorem 5 assures

$$
g^{p-1} A_{1}^{+}(g)\left(A_{1}^{-}(g)\right)^{1-p} \supset A_{p}^{+}(g) \cap g^{p}\left(A_{p}^{-}(g)\right)^{1-p} .
$$

But $A_{p}^{+}(g) \cap g^{p}\left(A_{p}^{-}(g)\right)^{1-p}=A_{p}^{+}(g)$, and this proves the factorization of a weight in $A_{p}^{+}(g)$.

Conversely, take $w_{0} \in A_{1}^{+}(g)$ and $w_{1} \in A_{1}^{-}(g)$, and let $w=g^{p-1} w_{0} w_{1}^{1-p}$. If $a \leq b \leq c$,

$$
\begin{aligned}
\int_{a}^{b} w\left(\int_{b}^{c} g^{p^{\prime}} w^{-1 / p-1}\right)^{p-1} \\
\quad=\int_{a}^{b} w_{0}\left(g^{-1} w_{1}\right)^{1-p}\left(\int_{b}^{c} w_{1}\left(g^{-1} w_{0}\right)^{1-p^{\prime}}\right)^{p-1} \\
\leq C\left(\int_{a}^{b} w_{0}(x)\left(\int_{x}^{x+h} g\right)^{p-1}\left(\int_{x}^{x+h} w_{1}\right)^{1-p} d x\right) \\
\quad \times\left(\int_{b}^{c} w_{1}(x)\left(\int_{x-s}^{x} g\right)^{p^{\prime}-1}\left(\int_{x-s}^{x} w_{0}\right)^{1-p^{\prime}} d x\right)^{p-1}
\end{aligned}
$$

for every $h, s>0$ by condition $A_{1}^{+}(g)$ for $w_{0}$ and $A_{1}^{-}(g)$ for $w_{1}$. In partic- 
ular, if $h=c-x$ and $s=x-a$ we obtain

$$
\begin{aligned}
\int_{a}^{b} w\left(\int_{b}^{c} g^{p^{\prime}} w^{-1 / p-1}\right)^{p-1} & \\
\leq & C\left(\int_{a}^{c} g\right)^{p}\left(\int_{a}^{b} w_{0}(x)\left(\int_{x}^{c} w_{1}\right)^{1-p} d x\right) \\
& \times\left(\int_{b}^{c} w_{1}(x)\left(\int_{a}^{x} w_{0}\right)^{1-p^{\prime}} d x\right)^{p-1} \\
& \leq C\left(\int_{a}^{c} g\right)^{p}\left(\int_{b}^{c} w_{1}\right)^{1-p}\left(\int_{a}^{b} w_{0}\right)\left(\int_{b}^{c} w_{1}\right)^{p-1}\left(\int_{a}^{b} w_{0}\right)^{\left(1-p^{\prime}\right)(p-1)} \\
& =C\left(\int_{a}^{c} g\right)^{p} .
\end{aligned}
$$

(C).

Theorem 6. If $w$ is in $A_{1}^{+}(g)$ then there exists $\delta>0$ such that

$$
\int_{a}^{b} g^{-\delta} w^{1+\delta}\left(\int_{a}^{b} g\right)^{-1} \leq C_{\delta} \int_{a}^{b} w\left(\int_{a}^{b} g\right)^{-\delta} g^{-\delta}(b) w^{\delta}(b)
$$

for every $a$ and a.e. $b$. For this $\delta, g^{-\delta} w^{1+\delta}$ is in $A_{1}^{+}(g)$.

Proof. Let $a$ and $b$ be real numbers with $a<b$ and with $b$ verifying

$$
M_{g}^{-}\left(g^{-1} w\right)(b) \leq C\left(g^{-1} w\right)(b)
$$

Let $O_{\lambda}=\left\{x: M_{g}^{-}\left(g^{-1} w \chi_{(a, b)}\right)(x)>\lambda\right\}$ be open. Then there exists a sequence of pairwise disjoint open intervals $I_{j}=\left(a_{j}, b_{j}\right)$ such that $O_{\lambda}=\bigcup I_{j}$ with

$$
\int_{a_{j}}^{x} w \chi_{(a, b)}\left(\int_{a_{j}}^{x} g\right)^{-1}>\lambda \quad \text { for every } x \in\left(a_{j}, b_{j}\right)
$$

and with

$$
\int_{a_{j}}^{b_{j}} w \chi_{(a, b)}\left(\int_{a_{j}}^{b_{j}} g\right)^{-1}=\lambda \quad \text { for every } j
$$

It is clear that each $a_{j}$ is bigger than $a$. Then, if $\lambda>C\left(g^{-1} w\right)(b)$, where $C$ is the $A_{1}^{+}(g)$ constant of $w$, each $I_{j}$ verifies either $(a, b) \supset I_{j}$ or $I_{j} \cap(a, b)=\varnothing$, since if $I_{j}$ is not contained in $(a, b)$ and $I_{j} \cap(a, b) \neq \varnothing$, then $b \in I_{j}$ and therefore

$$
\int_{a_{j}}^{b} w \chi_{(a, b)}\left(\int_{a_{j}}^{b} g\right)^{-1}>\lambda>C\left(g^{-1} w\right)(b)
$$

which goes against the election of $b$. 
By Lebesgue's differentiation theorem we have that $\left\{x \in(a, b):\left(g^{-1} w\right)(x)\right.$ $>\lambda\}$ is contained in $O_{\lambda}$. This relation, (7.3), and condition $A_{1}^{+}(g)$ for $w$ imply

$$
\int_{\left\{x \in(a, b):\left(g^{-1} w\right)(x)>\lambda\right\}} w \leq \lambda \sum_{\left\{j:(a, b) \supset I_{j}\right\}} \int_{I_{j}} g \leq \lambda \int_{\left\{x \in(a, b): C\left(g^{-1} w\right)(x)>\lambda\right\}} g .
$$

Let $\delta>0$. Multiplying the last inequalities by $\lambda^{\delta-1}$ and then integrating with respect to $\lambda$ from $C\left(g^{-1} w\right)(b)$ to $+\infty$ we get

$$
\begin{gathered}
\int_{C\left(g^{-1} w\right)(b)}^{+\infty} \lambda^{\delta-1}\left(\int_{\left\{x \in(a, b):\left(g^{-1} w\right)(x)>\lambda\right\}} w(x) d x\right) d \lambda \\
\leq C^{\delta+1}(1+\delta)^{-1} \int_{a}^{b}\left(g^{-1} w\right)^{1+\delta}(x) g(x) d x .
\end{gathered}
$$

On the other hand, the first item of (7.5) is equal to

$$
\begin{aligned}
& \int_{a}^{b}\left(\int_{C\left(g^{-1} w\right)(b)}^{\left(g^{-1} w\right)(x)} \lambda^{\delta-1} d \lambda\right) w(x) d x \\
& \quad=\delta^{-1} \int_{a}^{b} g^{-\delta} w^{1+\delta}-C^{\delta} \delta^{-1}\left(g^{-1} w\right)^{\delta}(b) \int_{a}^{b} w
\end{aligned}
$$

(7.5) together with (7.6) gives

$$
\left(\delta^{-1}-C^{1+\delta}(1+\delta)^{-1}\right) \int_{a}^{b} g^{-\delta} w^{1+\delta} \leq C^{\delta} \delta^{-1}\left(g^{-1} w\right)^{\delta}(b) \int_{a}^{b} w
$$

Choosing $\delta$ such that $\delta^{-1}-C^{1+\delta}(1+\delta)^{-1}>0$, we obtain the result.

Corollary 2. Let $1<p<\infty$. If $w$ is in $A_{p}^{+}(g)$ then there exists $\varepsilon>0$ such that $p-\varepsilon>1$ and $w$ is in $A_{p-\varepsilon}^{+}(g)$.

Proof. Let $w \in A_{p}^{+}(g)$. By factorization, there exist $w_{0}$ in $A_{1}^{+}(g)$ and $w_{1}$ in $A_{1}^{-}(g)$ such that $w=g^{p-1} w_{0} w_{1}^{1-p}$. By Theorem 6 there exist $\delta>0$ such that $g^{-\delta} w_{1}^{1+\delta} \in A_{1}^{+}(g)$. Then

$$
w=g^{p-1} w_{0} w_{1}^{1-p}=g^{p-\varepsilon-1} w_{0}\left(g^{-\delta} w_{1}^{1+\delta}\right)^{1-(p-\varepsilon)} \quad \text { with } \varepsilon=\delta(p-1)(1+\delta)^{-1}
$$

and the result follows from Corollary 1 .

Corollary 3. If $w \in A_{1}^{+}(g)$ then there exists $\gamma$ with $0<\gamma<1$, a function $k$ with $k$ and $k^{-1}$ in $L^{\infty}$, and a function $f$ such that $w=k g\left(M_{g}^{-} f\right)^{\gamma}$.

Proof. By Theorem 6, there exists $\delta>0$ such that $\left.M_{g}^{-}\left(g^{-1} w\right)^{1+\delta}\right)^{1 /(1+\delta)} \leq$ $C g^{-1} w$ a.e. On the other hand, Lebesgue's differentiation theorem gives $g^{-1} w$ $\leq\left(M_{g}^{-}\left(g^{-1} w\right)^{1+\delta}\right)^{1 /(1+\delta)}$. Let $k(x)=g^{-1}(x) w(x)\left(M_{g}^{-}\left(g^{-1} w\right)^{1+\delta}(x)\right)^{-1 /(1+\delta)}$. Then $C^{-1} \leq k \leq 1$ and $w=g k\left(M_{g}^{-} f\right)^{\gamma}$ where $\gamma=(1+\delta)^{-1}$ and $f=$ $\left(g^{-1} w\right)^{1+\delta}$. 
(D) Extrapolation. We can also state the following theorem.

Theorem 7. Let $T$ be a sublinear operator acting on measurable functions on R. Suppose that for a certain $p_{0}, 1 \leq p_{0}<\infty$, and for every $w$ in $A_{p_{0}}^{+}(g), T$ is of weak type $\left(p_{0}, p_{0}\right)$ with respect to the measure $w d x$. Then for every $p$ with $1<p<\infty$ and every $w$ in $A_{p}^{+}(g), T$ is bounded on $L^{p}(w d x)$.

The proof follows that of [3] with the obvious changes, which are essentially the definition of $G$ in Lemma 1 in [3] (now $\left.G=\left(g M_{g}^{-}\left(g^{-1} h^{1 / t} w\right) w^{-1}\right)^{t}\right)$ and the fact that $w$ is in $A_{p}^{+}(g)$ if and only if $g^{p^{\prime}} \sigma$ is in $A_{p^{\prime}}^{-}(g)$.

\section{REFERENCES}

1. K. Andersen and B. Muckenhoupt, Weighted weak type inequalities with applications to Hilbert transforms and maximal functions, Studia Math. 72 (1982), 9-26.

2. R. Coifman, P. W. Jones, and J. L. Rubio de Francia, Constructive decomposition of B.M.O. functions and factorization of $A_{p}$ weights, Proc. Amer. Math. Soc. 87 (1983), 675-676.

3. J. Garcia-Cuerva, An extrapolation theorem in the theory of $A_{p}$ weights, Proc. Amer. Math. Soc. 83 (1983), 422-426.

4. J. Garcia-Cuerva and J. L. Rubio de Francia, Weighted norm inequalities and related topics, North-Holland, Amsterdam, 1985.

5. B. Muckenhoupt, Weighted norm inequalities for the Hardy maximal function, Trans. Amer. Math. Soc. 165 (1972), 207-226.

6. E. Sawyer, A characterization of a two-weight norm inequality for maximal operators, Studia Math. 75 (1982), 1-11.

7. __, Weighted inequalities for the one-sided Hardy-Littlewood maximal functions, Trans. Amer. Math. Soc. 297 (1986), 53-61.

Departamento de Análisis Matemático, Facultad de Ciencias, Universidad de Málaga, 29071-Málaga, Spain (Current address of F. J. Martín-Reyes and A. de la Torre)

Current address (P. Ortega Salvador): Departamento de Estadistica y Econometria, Facultad de Economicas, Universidad de Malaga, 29013 Malaga, Spain 